\title{
Influence of Blood Flow Restriction Level on Muscle Fatigue During an Intermittent Isometric Exercise Taken to Failure
}

\author{
M. S. Cerqueira', R. Pereira ${ }^{2}$, G. Nunes de Mesquita ${ }^{1}$, T. Rocha ${ }^{1}$, A. Galvão de \\ Moura Filho' \\ ${ }^{1}$ Department of Physical Therapy, Federal University of Pernambuco, Pernambuco, Brazil \\ ${ }^{2}$ Department of Biological Sciences, State University of Southwest Bahia, Bahia, Brazil
}

\author{
CORRESPONDING AUTHOR: \\ Mikhail Santos Cerqueira \\ Department of Physical Therapy \\ Federal University of Pernambuco \\ University City \\ 50670-901 Recife \\ Pernambuco, Brazil \\ E-mail: mikalsantosc@hotmail.com
}

DOI:

10.32098/mltj.03.2020.08

LEVEL OF EVIDENCE: 1B

\begin{abstract}
SUMMARY
Background. Low-intensity exercise with blood flow restriction (BFR) may improve muscle mass and strength but an early neuromuscular fatigue may occur. Since the mechanisms of fatigue during BFR exercise is not yet fully understood we examined, concomitantly, the coefficient of variation of force (CVf) and surface electromyographic signal (sEMG) during an intermittent isometric handgrip exercise (IIHE) taken to failure.

Methods. 12 males completed an IIHE to failure with three blood flow conditions: total BFR (TR), partial BFR (PR) and free blood flow (FF). At each condition, three moments of the task were identified: beginning, midway and failure. The CVf and amplitude of rectified (arEMG) and median frequency (MDF) from the sEMG were analyzed.

Results. FF and PR conditions presented similar increase throughout the entire task in CVf $(\mathrm{FF}=1.73 \pm 0.88 \% ; \mathrm{PR}=1.61 \pm 0.63 \%)$ and $\operatorname{arEMG}(\mathrm{FF}=1.48 \pm 0.63 \% ; \mathrm{PR}=1.40 \pm 0.45 \%)$, and similar decrease in $\mathrm{MDF}(\mathrm{FF}=0.88 \pm 0.16 \% ; \mathrm{PR}=0.88 \pm 0.14 \%)$. Differently, during the task with TR there was a significant increase in CVf $(1.45 \pm 0.79 \%)$ and arEMG $(1.27 \pm 0.40 \%)$ and decrease in $\operatorname{MDF}(0.85 \pm 0.19 \%)$ only at the failure. The comparisons between conditions showed that the CVf, arEMG, and MDF were significantly lower $(p<0.05)$ at TR condition, when compared to the FF.

Conclusions. Simultaneous analysis of CVf and sEMG indicates that FF and PR conditions induce similar neuromuscular fatigue during an IIHE, while the adjustments to maintain the task are different in TR. Additionally, it does not seem necessary to add a partial BFR to increase muscle excitation during an exercise taken to failure.
\end{abstract}

\section{KEY WORDS}

Vascular occlusion; electromyography; motor control; median frequency; muscle activation; volitional fatigue.

\section{BACKGROUND}

Neuromuscular fatigue can be defined as a condition in which muscles fail to maintain a required effort (1). The mechanisms of exercise-induced muscle fatigue are related to the intensity, duration, type of muscle action and blood flow restriction (BFR) level $(2,3)$. Partial BFR, which have been widely investigated owing to its potential to improve muscle mass and strength at low-intensity training (4) is recognized as a condition that induces early neuromuscular fatigue (2). Poor arterial flow and venous stasis caused by insufflations of a pressure cuff in the proximal end of the active muscles can lead to muscle fatigue and trigger a number of mechanisms responsible for BFR training adaptations (5).

The maintenance of force output (obtained from straingauge/force transducer) during a fatiguing contraction is regulated by mechanisms as the recruitment of new motor units and the modulation of firing rates of active motor units (6). Some parameters related to the muscle features that have been used to investigate the control of force throughout fatiguing tasks are: (i) coefficient of variation of force $(\mathrm{CVf})$, that is influenced by the alterations in motor 
unit recruitment and/or discharge timing along the fatigue progresses; (ii) amplitude of the surface electromyographic signal (sEMG), that represents muscle excitation; (iii) median frequency (MDF) of sEMG spectrum, that is related to action potential conduction velocity $(6,7)$.

Increased muscle excitation appears to be of importance for strength gains (8) and hypertrophy (9), however the capacity of partial BFR to increase muscle recruitment is controversial (5). While some studies have shown higher neuromuscular excitation (10) others found no difference in the amplitude of sEMG during BFR exercise compared to free flow exercise with load-matched $(11,12)$. In addition, none of these studies investigated how different BFR levels influence neuromuscular activity during an isometric task taken to failure. Not least, it has been shown that MDF reduces during exercise with total BFR compared to free blood flow exercise (7), but the behavior of MDF during partial BFR exercise until failure is unclear.

Despite the potential relevance of CVf and sEMG assessments to verify how the neuromuscular activity/control is affected by BFR during a fatiguing task, no previous studies have simultaneously measured these outcomes. Additionally, considering that characteristics as blood pressure and limb circumference influences the restriction pressure (13), the arbitrary pressure generally applied $(7,11)$, may create a difference in the hypoxic intramuscular environment and hamper the understanding of mechanisms involved in the exercise-induced fatigue with different BFR levels. Therefore, this study aimed to compare the effects of different and individualized BFR levels on parameters of neuromuscular fatigue (CVf, sEMG amplitude and sEMG median frequency) during an isometric intermittent exercise taken to failure.

\section{MATERIALS AND METHODS}

\section{Participants}

Twelve healthy men (characteristics show in table I) classified as physically active or very active were included. They were right handed with no neuromuscular and cardiovascular disorders, and were naive to the all procedures. In the 24 hours antecedent to the experimental procedure participants avoided strenuous handgrip exercise and alcohol/ caffeine consumption. The same physical activity routine and diet were kept during the study period. Participants were informed of all experimental procedures as well as any potential risks associated with the study before giving written informed consent. This study is in accordance with Declaration of Helsinki and has also been previously approved by the Research Ethics Committee of the Feder-
Table I. Values are mean \pm standard deviation, minimum and maximum from subject characteristics $(n=12)$.

\begin{tabular}{lccc}
\hline Variable & Mean \pm SD & Minimum & Maximum \\
\hline Age $($ years $)$ & $21 \pm 1.71$ & 19 & 25 \\
\hline Height $(\mathrm{cm})$ & $177 \pm 6$ & 170 & 187 \\
\hline Body mass $(\mathrm{kg})$ & $78.58 \pm 9.50$ & 61.50 & 91.80 \\
\hline BMI $(\mathrm{kg} / \mathrm{m}-2)$ & $25.03 \pm 1.98$ & 21.28 & 28.47 \\
\hline SBP $(\mathrm{mmHg})$ & $120.15 \pm 11.60$ & 100 & 140 \\
\hline DBP $(\mathrm{mmHg})$ & $76.92 \pm 7.51$ & 70 & 90 \\
\hline TRP $(\mathrm{mmHg})$ & $126.92 \pm 10.52$ & 110 & 145 \\
\hline PRP $(\mathrm{mmHg})$ & $63.46 \pm 5.26$ & 55 & 72.5 \\
\hline
\end{tabular}

BMI, body mass index; SBP, systolic blood pressure; DBP, diastolic blood pressure; TRP, total restriction pressure; PRP, partial restriction pressure.

al University of Pernambuco under the protocol number CAAE: 36832814.9 .0000 .5208 . This trial was prospectively registered (Clinical Trials No. NCT02384161) and conducted ethically according to international standards of the journal as described by Padulo et al. (14).

\section{Experimental procedures}

Volunteers were submitted to three sessions of intermittent isometric handgrip exercise (IIHE) with three blood flow levels: total BFR (TR), partial BFR (PR) and free blood flow $(\mathrm{FF})$. The interval between two successive interventions ranged from $72 \mathrm{~h}$ (minimum interval) and 1 week (maximum interval). Thus, in this randomized crossover trial, all volunteers carried out the same IIHE sustained to failure with TR, PR and FF.

Through computer-generated random number tables (http://www.randomization.com/) the order of BFR level was allocated by an investigator who was not involved in the recruitment, intervention or assessment of subjects. Opaque sealed envelopes were used to conceal the allocation. To blind all procedures, the researcher responsible for maximum voluntary isometric contraction (MVIC) assessment and IIHE (researcher 1) did not know volunteer allocation. Evaluators determining (researcher 2) and applying/controlling restriction pressure (researcher 3 ) did not participate in the randomization. The volunteers were not informed about the BFR level applied and were instructed not to report the perception of pressure, keeping the researcher 1 blinded.

Initially, eligibility criteria were evaluated and anthropometric data were recorded. In the following day, blood pressure and the pressure necessary to total restriction (TRP - total restriction pressure) of brachial artery flow was determined, and the volunteers were familiarized with MVIC and IIHE. 
Then, $72 \mathrm{~h}$ after the familiarization, volunteers began the IIHE with different BFR conditions. Thenceforth, each IIHE session was identical, except for the BFR level. The volunteers were positioned in dorsal decubitus, with shoulder abducted at $90^{\circ}$, elbow fully extended and forearm supinated for all TRP and MVIC evaluations and during the IIHE.

\section{Total restriction pressure determination}

TRP of the brachial artery flow is a measure that shows good reproducibility and was determined according to the previous study (13). Briefly, the brachial artery blood flow of the dominant arm was detected by an ultrasound model SonoAceR3 (Samsung Medison - South Korea), by Power Doppler Technique - with $12 \mathrm{MHz}$ linear transducers placed in the flexor crease of the elbow. Visual and auditory signals indicated the presence of pulse during the cuff inflation (Aneroid sphygmomanometer Premium, Duque de Caxias - RJ; width cuff $14 \mathrm{~cm}$ ). The TRP was determined as the lowest pressure necessary to occlude completely the blood flow, and the partial occlusion pressure was set as the value corresponding to $50 \%$ of TOP. The cuff pressure in the FF condition was set as the pressure sufficient only to adjust to the arm $(\sim 10 \mathrm{mmHg})$, maintaining the forearm blood flow free. The same cuff used to determine the TRP (positioned at the dominant arm, just below the axilla near the insertion of the deltoid muscle) was inflated immediately before and deflated immediately after the end of IIHE.

\section{Force evaluation e fatiguing exercise}

Three MVIC of handgrip muscles were evaluated without any BFR and as previously described ${ }^{2}$. The recordings extracted from a custom-made strain gauge-based force transducer (DM 100, Miotec, Porto Alegre, RS, Brazil) were sampled at $2 \mathrm{kHz}$. The highest handgrip force of the three MVIC attempts was used to calculate the target force $(45 \%$ of MVIC) throughout the IIHE. Five minutes after the third MVIC, the volunteers started the IIHE, carrying out successive isometric handgrip contractions, each of one sustained for 10 seconds, followed by 5 -seconds resting, until the failure. Volunteers were instructed to reach and maintain the target as brief and accurately as possible during active phase and relax (force $=0$ ) during the resting. The failure was defined as the incapacity to reach and sustain a force greater than $30 \%$ of MVIC for 5 seconds or more in three consecutive contractions (15). The periods of action and resting were controlled by a metronome-timed beep sound. Throughout the entire task, the force was displayed by projection on the ceiling located approximately two meters away from the volunteer. Volunteers received strong verbal encouragement from researcher 1 during the MVIC assessments and along all the IIHE.

\section{Surface EMG recordings}

sEMG were obtained using four channel modules (Miotool 400, Miotec Equipment, Biomedical, Brazil) with a total amplifier gain of 1000, a common mode rejection ratio of $110 \mathrm{~dB}$, sampled at $2.0 \mathrm{kHz}$ and band-pass filtered $(5-500$ $\mathrm{Hz}$ ). A 14-bit converter digitalized the analog signals with a sampling frequency of anti-aliasing $1.0 \mathrm{kHz}$ for each channel. Pre-amplified (x100) bipolar superficial and circular adhesive electrodes of $\mathrm{Ag} / \mathrm{AgCl}$ (teardrop shape and diameter $3 \mathrm{~cm}$ ) were used with interelectrode (center-to-center) distance of $20 \mathrm{~mm}$. The skin was shaved and cleaned with alcohol, and anatomical landmarks (proximal third of the forearm, between the styloid processes of the radius and the humeral medial epicondyle) were determined to place electrodes over the flexor digitorum superficialis forearm and flexor carpi radialis (16). The reference electrode $(\mathrm{Ag} / \mathrm{AgCl}$, oval shaped, $5 \times 3.8 \mathrm{~cm}$ diameter $)$ was placed just below the sternal notch. Since the surface electrodes mainly detect the EMG signal of the muscle beneath, some activity from nearby muscles could also be picked up. Thus, for this reason, and because various forearm muscles flexing the carpus and the fingers, we ascribed the signal from the electrodes over the cited muscles as forearm flexor muscles (17). All described procedures followed the recommendation of SENIAM (Surface ElectroMyoGraphy for Non-Invasive Assessment of Muscles) project (16).

\section{DATA ANALYSIS}

Force and sEMG signals were recorded continuously and analyzed simultaneously at the beginning, midway and failure moments of the task. The first contraction was excluded by generally be atypical owing to the task adaptation, thus the next contraction was considered the first valid contraction (beginning), that was used to normalize the others time point measures. Task failure was defined as the incapacity to keep the force of at least $30 \%$ of MVIC in two consecutive contractions. Then, the last valid contraction was defined as the contraction immediately before the first of the two consecutive failures contractions. The midway of the task was defined as the contraction that divided in half the total number of valid repetitions. The epochs were identified based on force signals from visual inspection, and EMG was segmented based on force onsets-offsets. A specific algorithm was applied to analyze 6 seconds from each contraction, which was lasted for 10 seconds. For then, the first and 
the last 2 seconds were excluded. This procedure guarantee that $\sim 60 \%$ of the action phase were analyzed, excluding the adjusting of force to reach the target force, at the first 2 seconds, and the last two seconds were the motor control was more imprecise, since the finish of active phase is close. The force data was band passed filtered between 0.05 and $15 \mathrm{~Hz}$ (Butterworth, $4^{\text {th }}$ order) and detrended before motor output variability measures were calculated. The within-trial variability was measured using the standard deviation (SD) of the detrended filtered force and as the CVf (SD of force/mean force) x100], as done by Pereira et al. (2012) (17). sEMG was studied in the time [amplitude of rectified sEMG (arEMG)] and frequency (median frequency [MDF] of sEMG) domains. For arEMG analysis the sEMG was band passed filtered between 5 and $500 \mathrm{~Hz}$ (Butterworth, $4^{\text {th }}$ order), and then, full-wave rectified. The Fast Fourier transform (FFT - Hamming window processing) was applied to the raw sEMG to identify the MDF of the power spectrum periodogram. The data from CVf, arEMG and MDF obtained at midway and failure moments were normalized by the beginning of the task. Then, the results are reported as a percentage (\%) of the beginning.

\section{STATISTICAL ANALYSIS}

The data distributions of normality were evaluated by the Kolmogorov-Smirnov test. A two-way ANOVA (3 blood flow levels $\times 3$ moments throughout the task) was used to compare the CVf, arEMG and MDF. Significant ANOVA results were followed by appropriate post hoc tests with Bonferroni corrections. The level of significance was set to $p<0.05$ and the analyses were performed with the SPSS17.0 statistical package (SPSS Inc., Chicago, IL. USA). Data are reported as means $\pm \mathrm{SD}$ in the text and tables and means $\pm \mathrm{SE}$ (standard error) in the figures. Only the normalized data (midway or failure /beginning) were considered in the statistical analysis. The non-normalized data obtained before (to verify similarity of initial values between the evaluation days) and during exercise are shown in table II. The number of subjects included was determined based upon previous BFR investigations that observed similar outcome measures $(7,12)$.

\section{RESULTS}

The analyses of CVf exhibited a significant main effect for measures $(F$-value $=30.7, \mathrm{p}<0.001)$. The post-hoc comparisons indicated that FF and PR conditions exhibited a similar behavior increasing significantly at the midway of the task $(\mathrm{FF}=1.94 \pm 0.78 \% ; \mathrm{PR}=1.76 \pm 0.64 \%)$ and at the failure $(\mathrm{FF}=2.26 \pm 0.97 \%$; $\mathrm{PR}=2.07 \pm 0.44 \%)$, while in the $\mathrm{TR}$

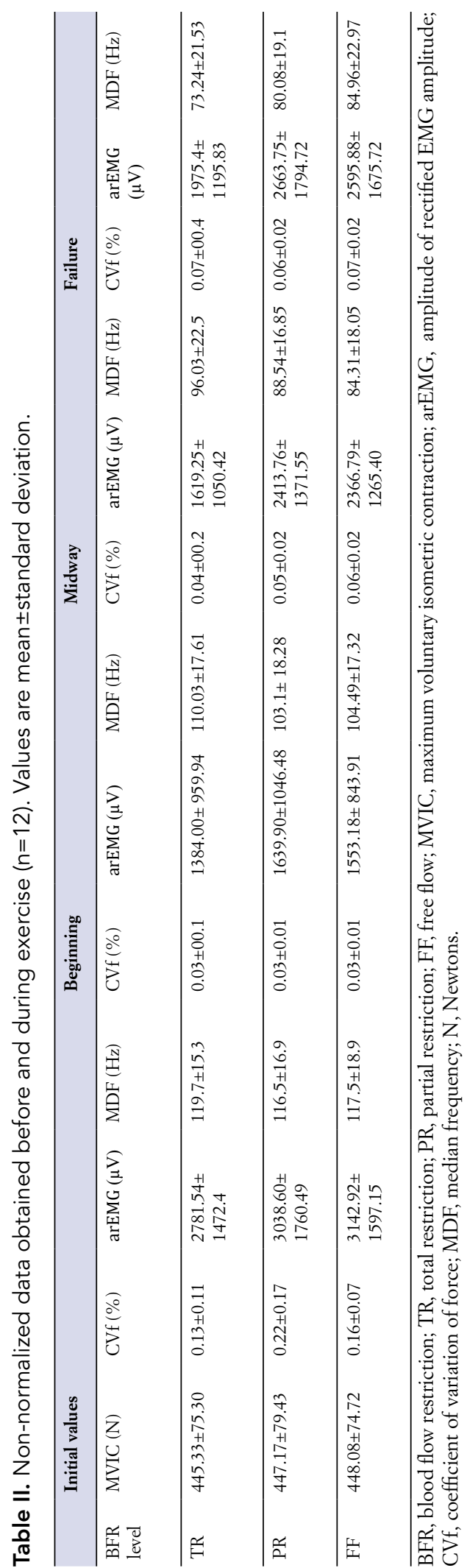

Muscles, Ligaments and Tendons Journal 2020;10 (3) 
condition the CVf increased significantly $(2.09 \pm 0.97 \%)$ only at the task failure. Additionally, the CVf was significantly greater in FF $(1.94 \pm 0.78 \%)$ condition at midway of the task, when compared to the TR $(1.26 \pm 0.58 \%$ (figure 1 ). The arEMG also exhibited a significant main effect for measures $(\mathrm{F}$-value $=24.3, \mathrm{p}<0.001)$, with $\mathrm{FF}$ (midway $=$ $1.66 \pm 0.55 \%$; task failure $=1.76 \pm 0.76 \%$ ) and PR (midway $=1.56 \pm 0.36 \%$; task failure $=1.65 \pm 0.49 \%$ ) conditions increasing significantly at midway and task failure moments, when compared to the beginning (figure 2). However, at the TR, the arEMG increased significantly only at the failure $(1.57 \pm 0.45 \%)$, when compared to the beginning. As observed for CVf, arEMG was significantly greater in the FF $(1.66 \pm 0.55 \%)$ condition at midway of the task, when compared to TR $(1.23 \pm 0.34 \%)$. For the MDF analyses (figure 3), it was also observed a significant main effect for measures $(\mathrm{F}$-value $=35.6, \mathrm{p}<0.001)$. The FF and PR conditions exhibited similar behavior decreasing significantly at the midway ( $\mathrm{FF}=0.82 \pm 0.16 \% ; \mathrm{PR}=0.86 \pm 0.12 \%)$ and at failure $(\mathrm{FF}=0.81 \pm 0.16 \%$; $\mathrm{PR}=0.78 \pm 0.15 \%)$ moments, while the TR decreased significantly only at the failure $(0.66 \pm 0.13 \%)$. Additionally, MDF was also significantly smaller in FF condition $(\mathrm{FF}=0.81 \pm 0.16 \%)$ at the failure, when compared to TR $(0.66 \pm 0.13 \%)$.

Regarding to the reliability of the measures, intra-reliability for CV values ranged from 3.7 to $4.7 \%$ for CIVM, 8.7 to $11.7 \%$ for MDF, and 10.4 to $12.9 \%$ for arEMG. Inter-reliability for CV values were $4.4 \%$ for CIVM, $10.8 \%$ for MDF and $26.1 \%$ for arEMG.

\section{DISCUSSION}

Our main findings were: (i) throughout the task, the FF and $\mathrm{PR}$ conditions induced similar increases in CVf and arEMG, as well as similar decreases in MDF; (ii) the TR condition exhibited significant increases in CVf and arEMG, as well as decreases in MDF, only at the failure; (iii) CVf, arEMG and MDF were significantly lower in the TR condition, only when compared to FF.

The CVf is a motor output measure, indicating that force during voluntary actions fluctuates around a target (17), however, CVf have not been studied during BFR exercises. CVf along a submaximal fatiguing task is influenced by decreased cortical excitability and motoneuron discharge, inhibition by Renshaw cells, and type III and IV afferents or decreased excitation of Ia afferents (18). Our results indicated that the motor output was similar between PR and FF conditions, increasing constantly throughout the entire task, while the TR induces a significant increase only at the failure. In our study, the typical metabolites accumulation imposed by the BFR was not sufficient to induce differ-

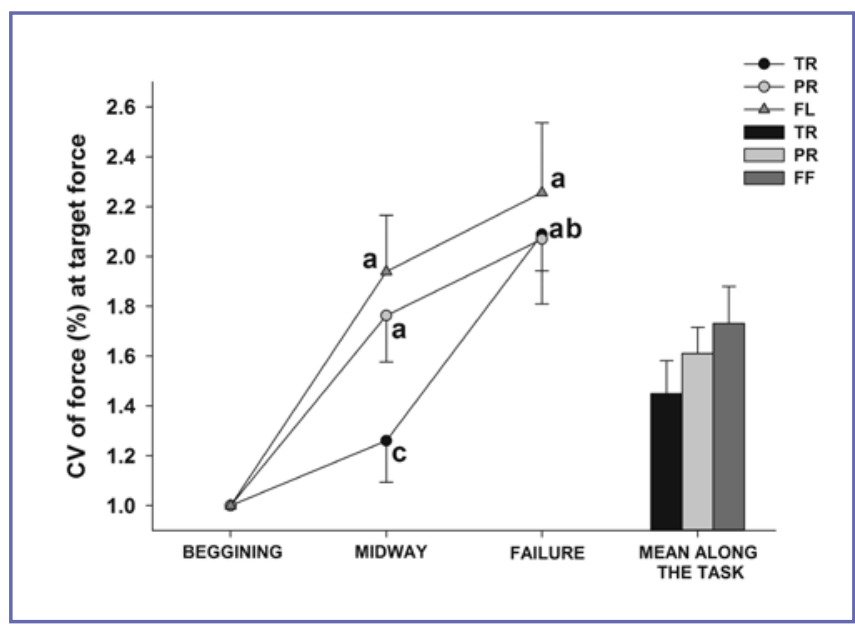

Figure 1. Mean \pm SE of CV of force (\%) at the beginning, midway and failure, as well as the mean along the task. Data are presented for total restriction (TR), partial restriction (PR) and free flow (FF). a: significantly different from the beginning of task $(p<0.05)$; b: significantly different from beginning and midway of task in the TR condition $(p<0.05)$; $c$ : significantly different from FF condition at the midway of task $(p<0.05)$.

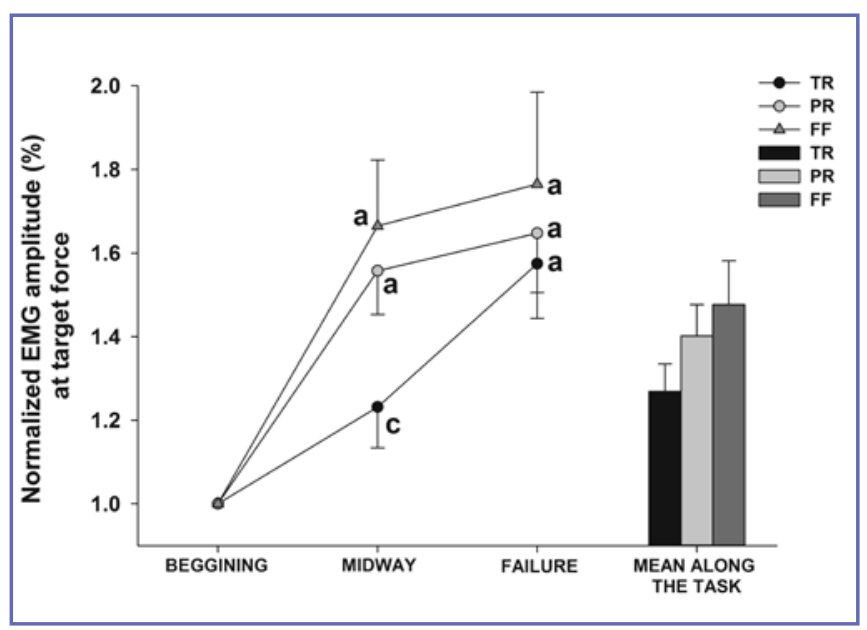

Figure 2. Mean \pm SE of normalized $E M G$ amplitude (\%) at the beginning, midway and failure, as well as the mean along the task. Data are presented for total restriction (TR), partial restriction (PR) and free flow (FF). a: significantly different from the beginning of task $(p<0.05)$; $c$ : significantly different from free flow (FF) condition at the midway of task $(p<0.05)$.

ent sensoriomotor integration between PR and FF conditions. On the other hand, TR induces divergent sensoriomotor integration from the FF condition, since the CVf did not increase at the midway of the task. A sudden increase in CVf may indicate a facilitation of force, which may be 


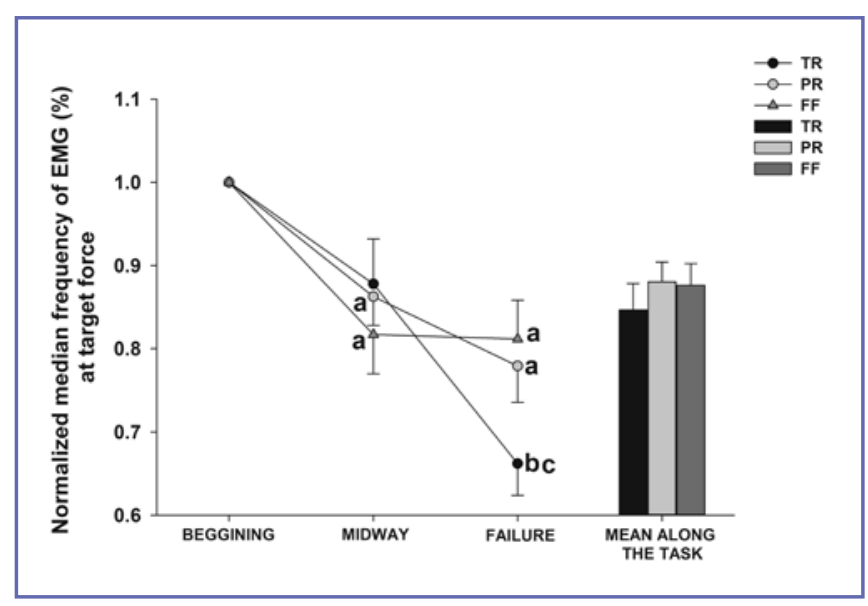

Figure 3. Mean \pm SE of normalized median frequency of EMG signal (\%) at the beginning, midway and failure, as well as the mean along the task. Data are presented for total restriction (TR), partial restriction (PR) and free flow (FF). a: significantly different from the beginning of task $(p<0.05)$; $b$ : significantly different from beginning and midway of task in the TR condition $(p<0.05)$; $c$ : significantly different from free flow (FF) condition at the failure $(p<0.05)$.

sustained by augmented motor unit recruitment, increases and decreases in rate coding, alterations in motor control, and neural and post activation potentiation (18). Thus, the behavior adopted during the TR condition could indicate a strategy to maintain the task, delaying the motor unit recruitment increases, probably as a way of saving energy in a situation of extreme oxygen shortage. Interestingly the PR, despite the imposed blood flow limitation, was not sufficient to change the neuromuscular strategy than FF condition, suggesting that differences in the long term exercise adaptations induced by a PR could be related to the molecular level, than to the neuromuscular level.

The results from sEMG corroborate our hypothesis raised from the CVf. In other words, if an increasing in the descending drive along the task is necessary to maintain the force, an increase in sEMG amplitude, as well as a decrease in MDF should be expected, owing to an increasing in discharge rates of active motor units and/or adding motor units recruitment (19). In fact, we identified a significant and similar increase in arEMG throughout the task in the $\mathrm{FF}$ and PR. This is in agreement with previous results indicating that increases in sEMG amplitude has been associated to the increases in CVf (19). Accordingly, it is suggested, that the sudden increases in CVf can be partially explained by the recruitment of additional motor units to compensate the decline in force capacity of the already activated motor units by recruiting additional higher threshold motor units
(20). In fact, sudden increases of arEMG in the TR condition at the failure could be attributable to enhanced excitatory descending drive onto the motoneuron pool, as also suggested by the more rapid increases in CVf (21).

Although PR has been shown to augment arEMG over FF with load-matched protocols $(10,22)$, we found a similar increase in arEMG during FF and PR conditions. Our findings agree with previous studies ${ }^{11,12}$ that showed no difference between FF and PR conditions when low-intensity exercise was conducted until failure. Thus, the combined findings of these previous investigations and the current study findings constitute robust evidence indicating that, when taken a submaximal task to failure, PR induces similar neuromuscular strategies than FF condition.

On the other hand, it has been suggested that higher occlusion pressure increases neural compression and energetic demand, resulting in the elevated neuromuscular excitation commonly observed with BFR exercise $(7,23)$. However, we observed an attenuated arEMG rise at the midway during the task with TR. This disagreement may be due to the fact that in previous studies the exercise did not reach failure and was carried out at smaller load (20\% of 1-RM or MVIC). Another explanation for this attenuated rise of arEMG in TR condition is that the metabolite accumulation (sensed by type III and IV muscle afferents) may inhibit alpha motor neurons and/or the descending neural drive, mitigating the motor unit recruitment and reducing muscle excitation (24).

As observed for CVf and arEMG, the behavior of MDF in FF and PR conditions was similar, with a drastic decrease from the beginning to the midway of the task and sustained until the failure. In TR condition there was a little decrease at midway of the task with an abrupt and significant reduction at failure, when compared to the beginning and midway of the task. Again, as observed for CVf and arEMG, significant difference was observed only between FF and TR conditions, but now, at the failure moment. These results agree with previous results that did not observed differences in spectral parameters of the sEMG during low-intensity exercise with PR or FF (25). In addition, the greater MDF decrease in the TR condition, when compared to FF, was also observed in previous studies $(7,22)$. It is important to note that higher decrements of median frequency are related to higher force fluctuations (26), indicating once again the consistency of our results among all evaluated variables. Under fatigue conditions, the motor units synchronization and fibers conduction velocity impairment cause spectral shift towards low-frequency regions (27). Thus, at first glance it may appear that the greater MDF decrease at task failure, as observed during the TR condition, indicates greater fatigue, which could be supported by the statement 
that higher occlusion pressures may cause greater magnitude of muscle fatigue (28). It is noteworthy, however, that despite precipitate the muscle failure, the same exercise protocol with TR allows a faster force recovery after the task failure, when compared to FF and PR (2). It is reasonable to consider, therefore that TR can cause selective fatigue in the oxygen-independent muscle fibers, thus contributing to a depletion of the glycolytic fibers, greater accumulation of $\mathrm{H}^{+}$and consequently greater reduction in MDF (7). Then, from the reestablishment of blood flow, the partially fresh oxygen-dependent fibers would contribute to the faster return of the force-producing capacity. In FF and PR conditions, however, both glycolytic and oxidative fibers were likely to fatigue, causing a greater magnitude of function impairment as indicated by a slower recovery in the capacity of force production post-failure. In short, considering previous data on force recovery post-failure and the behavior of the CVf and arEMG found in the present study, the sudden MDF reduction at failure in the TR condition does not indicate a greater magnitude of fatigue, but rather a specific strategy to sustain the task for a long time as possible in a metabolically unfavorable condition.

\section{Limitations}

The current study is not free of limitations. First, the IIHE protocol used differs from most studies in which dynamic contractions are performed by larger (knee extensors and elbow flexors) muscle groups. However, to add the CVf in the muscle fatigue analysis, we chose to work with the wrist and finger flexors, enabling the maintenance in the target force due to higher motor control of these muscles (17). Moreover, isometric actions minimize some methodological limitations related to the sEMG recorded during dynamic actions (29). Additionally, we did not include a high intensity exercise with free flow condition. Recent systematic review, however, indicate that partial BFR only potentiates strength gains when compared to the low-in-

\section{REFERENCES}

1. Boyas S, Guével A. Neuromuscular fatigue in healthy muscle: underlying factors and adaptation mechanisms. Ann Phys Rehabil Med 2011;54(2):88-108. doi:10.1016/j.rehab.2011.01.001

2. Cerqueira MS, Pereira R, Rocha T, et al. Time to failure and neuromuscular response to intermittent isometric exercise at different levels of vascular occlusion: a randomized crossover study. Int J Appl Exer Physiol 2017;6(1):55-70. doi:10.22631/ ijaep.v6i1.108.

3. Babault N, Desbrosses K, Fabre M-S, Michaut A, Pousson M. Neuromuscular fatigue development during maximal concen- tensity exercise with FF, but not in comparison to the high intensity exercise (30). Lastly, we do not provide measures of the blood perfusion/oxygenation in the muscle tissue (e.g. near infrared spectroscopy). Considering that $50 \%$ of blood restriction used in the present study does not necessarily mean $50 \%$ of oxygenation level/perfusion reduction, measurement of muscle perfusion /oxygenation could help explain the absence of difference between FF and PR conditions in the mechanical and electrophysiological variables.

\section{Practical implications}

Despite augmented muscle excitation is considered as a potential mechanism underlying strength gains and hypertrophy conducted by low-intensity BFR training the literature involving sEMG parameters is discordant. In this sense, our results summed to previous indicate that, when executed until task failure, the PR induces similar neuromuscular strategies than FF. From a practical viewpoint, this finding supports that subjects with contraindications for tourniquet use may obtain stimulus for muscular adaptation when exercise is taken to failure even without partial BFR.

\section{CONCLUSIONS}

Our results indicate, from a simultaneous analysis of CVf, arEMG and MDF during a submaximal intermittent isometric exercise, that the FF and PR require similar neuromuscular strategies to carry out the task until the failure and that TR induce different neuromuscular adjusts to maintain the task. Additionally, we suggest that it seems not necessary to add a partial BFR to increase muscle excitation during an exercise taken to failure.

\section{CONFLICT OF INTERESTS}

The authors declare that they have no conflict of interests.

tric and isometric knee extensions. J Appl Physiol 2006;100(3): 780-785. doi:10.1152/japplphysiol.00737.2005.

4. Patterson SD, Hughes L, Warmington S, Burr JF, Scott BR, Owens J, et al. Blood Flow Restriction Exercise: Considerations of Methodology, Application, and Safety. Front Physiol 2019;10:533. doi:10.3389/FPHYS.2019.00533.

5. Pearson SJ, Hussain SR. A review on the mechanisms of bloodflow restriction resistance training-induced muscle hypertrophy. Sports Med 2015;45(2):187-200. doi:10.1007/s40279. 014-0264-9. 
6. Contessa P, Adam A, De Luca CJ. Motor unit control and force fluctuation during fatigue. J Appl Physiol 2009; 107(1):235-43 doi:10.1152/japplphysiol.00035.2009.

7. Moritani T, Sherman WM, Shibata M, Matsumoto T, Shinohara $M$. Oxygen availability and motor unit activity in humans. Eur J Appl Physiol Occup Physiol 1992;64(6):552-6.

8. Shinohara M, Kouzaki M, Yoshihisa T, Fukunaga T. Efficacy of tourniquet ischemia for strength training with low resistance. Eur J Appl Physiol Occup Physiol 1998;77(1-2):189-91.

9. Morton RW, Oikawa SY, Wavell CG, Mazara N, McGlory C, Quadrilatero J, et al. Neither load nor systemic hormones determine resistance training-mediated hypertrophy or strength gains in resistance-trained young men. J Appl Physiol 2016;121(1):129-138. doi:10.1152/japplphysiol.00154.2016.

10. Killinger B, Lauver JD, Donovan L, Goetschius J. The Effects of Blood Flow Restriction on Muscle Activation and Hypoxia in Individuals with Chronic Ankle Instability. J Sport Rehabil 2019;16:1-25 doi:10.1123/jsr.2018-0416.

11. Wernbom M, Järrebring R, Andreasson MA, Augustsson J. Acute effects of blood flow restriction on muscle activity and endurance during fatiguing dynamic knee extensions at low load. J Strength Cond Res 2009;23(8):2389-2395. doi:10.1519/ JSC.0b013e3181bc1c2a.

12. Cayot TE, Lauver JD, Silette CR, Scheuermann BW. Effects of blood flow restriction duration on muscle activation and microvascular oxygenation during low-volume isometric exercise. Clin Physiol Funct Imaging 2016;36(4)298-305. doi:10.1111/ cpf.12228.

13. Bezerra de Morais AT, Cerqueira MS, Moreira Sales R, Rocha T, Moura Filho AG. Upper limbs total occlusion pressure assessment: Doppler ultrasound reproducibility and determination of predictive variables. Clin Physiol Funct Imaging 2017;37(4):437-441. doi:10.1111/cpf.12330.

14. Padulo J, Oliva F, Frizziero A, Maffulli N. Muscles, Ligaments and Tendons J - Basic principles and recommendations in clinical and field science research: 2016 update.MLTJ 2016;6(1):15. 10.11138/mltj/2016.6.1.001.

15. Cerqueira MS, Pereira R, Mesquita GN, Rocha T, Moura Filho AG. Rate of force development to evaluate the neuromuscular fatigue and recovery after an intermittent isometric handgrip task with different blood flow restriction conditions. Motriz 2019;25(1):1-6. doi:10.1590/s1980-6574201900010009.

16. Hermens HJ, Freriks B, Disselhorst-Klug C, Rau G. Development of recommendations for SEMG sensors and sensor placement procedures. J Electromyogr and Kinesiol 2000(5);10:361374. doi:10.1016/S1050-6411(00)00027-4.

17. 17. Pereira R, Freire IV, Cavalcanti CVG, Luz CPN, Neto OP. Hand dominance during constant force isometric contractions: evidence of different cortical drive commands. Eur J Appl Physiol 2012(8);112: 2999-3006. doi:10.1007/s00421-011-2278-4.

18. Behm DG. Force maintenance with submaximal fatiguing contractions. Can J Appl Physiol 2004;29(3):274-90.
19. Mendez-Villanueva A, Baudry S, Riley ZA, Rudroff T. Influence of rest duration on muscle activation during submaximal intermittent contractions with the elbow flexor muscles. J Sports Med Phys Fitness 2009;49(3):255-64.

20. Löscher WN, Cresswell AG, Thorstensson A. Excitatory drive to the $\alpha$-motoneuron pool during a fatiguing submaximal contraction in man. J Physiol 15():271-80. doi: 10.1113/jphysiol.1996.sp021214.

21. Carson RG, Riek S, Shahbazpour N. Central and peripheral mediation of human force sensation following eccentric or concentric contractions. J Physiol 2002;15(Pt3):913-25. doi:10.1113/jphysiol.2001.013385.

22. Yasuda T, Brechue WF, Fujita T, Shirakawa J, Sato Y, Abe T. Muscle activation during low-intensity muscle contractions with restricted blood flow. J Sports Sci. 2009;27(5):479-89. doi:10.1080/02640410802626567

23. Yasuda T, Brechue WF, Fujita T, Sato Y, Abe T. Muscle activation during low-intwnsity muscle contraction with varying levels of external limb compression. J Sports Sci Med 2008;7(4):467-474.

24. Light AR, Hughen RW, Zhang J, Rainier J, Liu Z, Lee J. Dorsal Root Ganglion Neurons Innervating Skeletal Muscle Respond to Physiological Combinations of Protons, ATP, and Lactate Mediated by ASIC, P2X, and TRPV1. J Neurophysiol 2008;100(3):1184-1201. doi:10.1152/jn.01344.2007.

25. Karabulut M, Cramer JT, Abe T, Sato Y, Bemben MG. Neuromuscular fatigue following low-intensity dynamic exercise with externally applied vascular restriction. J Electromyogr and Kinesiol 2010;20(3):440-447. doi:10.1016/j.jelekin.2009.06.005.

26. Kouzaki M, Shinohara M, Masani K, Fukunaga T. Force fluctuations are modulated by alternate muscle activity of knee extensor synergists during low-level sustained contraction. J Appl Physiol 2004;97(6):2121-2131. doi:10.1152/japplphysiol.00418.2004.

27. Bigland-Ritchie B, Donovan EF, Roussos CS. Conduction velocity and EMG power spectrum changes in fatigue of sustained maximal efforts. J Appl Physiol 1981;51(5):1300 1305. doi:10.1152/jappl.1981.51.5.1300.

28. Fatela P, Reis JF, Mendonca G V, Avela J, Mil-Homens P. Acute effects of exercise under different levels of blood-flow restriction on muscle activation and fatigue. Eur J Appl Physiol 2016;116(5):985-95. doi:10.1007/s00421-016-3359-1.

29. González-Izal M, Malanda A, Gorostiaga E, Izquierdo M. Electromyographic models to assess muscle fatigue. J Electromyogr and Kinesiol 2012;22(4):501-512. doi:10.1016/j.jelekin.2012.02.019.

30. Centner C, Wiegel P, Gollhofer A, König D. Effects of Blood Flow Restriction Training on Muscular Strength and Hypertrophy in Older Individuals: A Systematic Review and Meta-Analysis. Sports Med 2019;49(1):95-108. doi:10.1007/s40279-0180994-1. 